\title{
Simulation of Quasi-Static Folding and Deployment of Ultra-Thin Composite Structures
}

\author{
H.M.Y.C. Mallikarachchi* \\ University of Cambridge, Cambridge, CB2 1PZ, UK. \\ S. Pellegrino ${ }^{\dagger}$ \\ California Institute of Technology, Pasadena, CA 91125
}

\begin{abstract}
This paper presents a detailed study of the folding and deployment of a slotted tube hinge made from a two-ply laminate of carbon-fibre reinforced plastic. A physical model of a particular version of this hinge has been used to carry out quasi-static deployment tests and this process has been fully captured through a finite element simulation. The first stage in this simulation was to generate the fully folded, strained configuration of the hinge. The second stage in the simulation was to gradually decrease the relative rotation between the ends until it became zero. By analysing the in-plane strains and out-of-plane curvatures in the folded configuration we have confirmed that the particular hinge design that has been studied could be folded without permanent damage.
\end{abstract}

\section{Introduction}

Stored energy deployable structures made from flat or curved sheets of ultra-thin composites have already been used for a number of missions and are currently being considered for several new applications. Their main attractions are their light weight and low cost, due to the small number of component parts. Examples of structures of this kind that have been already flown include the Boeing Springback reflectors on the MSAT and TDRS H, I, J spacecraft and the three NGST Astro Aerospace Foldable Flattenable Booms forming the Mars Advanced Radar for Subsurface and Ionosphere Sounding (MARSIS) antenna on the Mars Express spacecraft. ${ }^{1}$ Several novel solar array and antenna concepts based on the same general approach have already been proposed, including the "hollow solid" reflector structure ${ }^{2}$ shown in Figure 1, the Fold Integrated Thin-film Stiffener (FITS) solar array, which undergoes three different folding stages namely root fold, z-fold and tri-fold to make a compact structure, ${ }^{3}$ and the Folding Large Antenna Tape Spring (FLATS) radar concept. ${ }^{4}$

Although the deployment behavior of these structures may appear to be simple, as they seem to have a natural tendency towards springing open and thus reach a configuration with zero strain energy, in reality these structures have to be designed carefully in order to avoid permanent deformation or fracture of the material. This process is made more complex by the ease with which an elastically strained structure can change configuration and find a lower energy state - which may well be associated with much higher strains in the material than any expected configurations - that had not been identified by the designer. Indeed, it is just as important to fully understand and control the folding of the structure, because the same problems can be encountered also at this stage. An example of unexpected behavior occurred during the deployment of the first MARSIS boom. ${ }^{5}$ The difficulty of carrying out reliable ground deployment tests on these structures should also be noted.

A substantial research effort will be required to fully address this situation. The simulations will need to capture the large-displacement behavior of each component and particularly any localized deformation features of these ultra-thin structures, including the effects of contact between different parts. Non-linearity

\footnotetext{
*Research Student, Department of Engineering, Trumpington Street

${ }^{\dagger}$ Professor of Aeronautics and Civil Engineering, Graduate Aeronautical Laboratories, 1200 East California Boulevard, Mail Code 301-46, Fellow AIAA. e-mail: sergiop@caltech.edu
} 

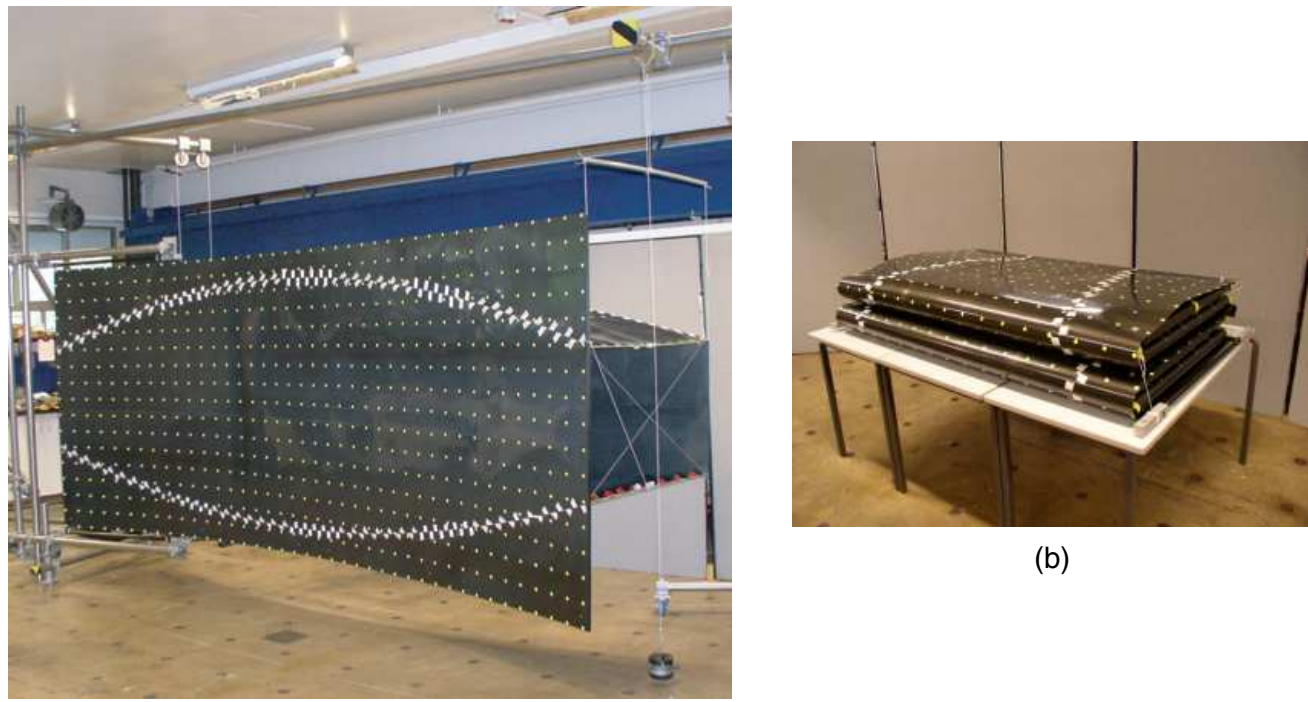

(b)

(a)

Figure 1. Hollow solid reflector (a) deployed and (b) folded.

in the behavior of the material will also have to be included in the models. The experiments will need to address gravity effects on lightweight structures of realistic complexity and scale.

This paper presents an initial step towards achieving these aims. We consider a simple foldable structure that poses many of the challenges that will be encountered in larger structures, but this particular example is itself of significant practical interest, as there are a number of applications where it could be used. This structure is known as a "tube hinge" and consists of a thin-walled tube made of carbon fibre reinforced plastic with two longitudinal slots with round ends, as shown in Figure 2. A variant of this tube hinge design, with three slots, was considered by Yee and Pellegrino; ${ }^{6}$ also each folding section of the MARSIS booms is in fact a tube with two slots with enlarged round ends.

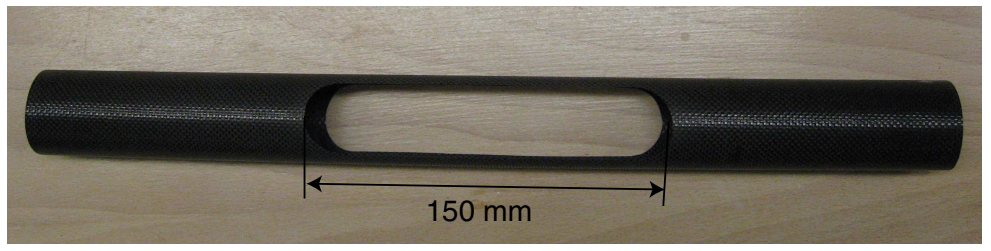

Figure 2. Top view of tube hinge (deployed).

We present a study of a particular tube hinge design for which we have carried out both quasi-static deployment tests using a bending rig as well as folding and deployment simulations with the commercial finite element package ABAQUS/Explicit. ${ }^{7}$ Section II presents the deployment experiments that were carried out. Section III presents the linear-elastic material model that is adopted for the woven composite; this takes the form of a $6 \times 6 \mathrm{ABD}$ matrix that is derived from homogenization theory. Sections IV and V present the folding and deployment simulations for the tube hinge and include sensitivity studies that are used to assess the accuracy of the fully folded configuration that is achieved. This configuration provides the starting point for the deployment simulation and is also the most highly deformed configuration of the structure. The in-plane strains and out-of-plane curvatures, from which the margin of safety against failure can be evaluated, are presented in Section VI. Section VII presents a comparison between a deployment simulation and the experimental results. Section VIII concludes the paper. 


\section{Experiments}

The tube hinge used for the experiment has an internal diameter of $38 \mathrm{~mm}$ and thickness of $0.2 \mathrm{~mm}$. It consists of a two-ply $[ \pm 45]_{2}$ plain weave laminate of $1 \mathrm{k}$ tows of T300 carbon fibres impregnated with Hexcel 913 epoxy resin. The tube was manufactured by laying two plies of resin-impreganted fabric on a $38 \mathrm{~mm}$ diameter steel tube sprayed with PTFE release agent. The fabric is wrapped in PTFE release film and is held by a heat-shrinking sleeve. The tube was cured under vacuum for one hour at $125^{\circ}$ and $600 \mathrm{kPa}$. After cooling, the steel tube was pulled out and then the two parallel slots were cut with a pressurized water jet machine. $^{8}$

The folding section of the tube hinge includes two $150 \mathrm{~mm}$ long slots consisting of a $120 \mathrm{~mm}$ rectangular cut with $15 \mathrm{~mm}$ diameter half circles at the ends, Figure 2.

Folding of the hinge is best done by pinching it in the middle and then, having flattened the central cross section, bending the already softened structure by rotating the ends of the hinge in opposite directions. This avoids applying high bending moments that may damage the hinge. It will be shown in Section IV that the bending moment required to buckle the straight and unflattened hinge is more than double the peak moment that occurs during deployment; it is unnecessary to apply such a large moment.

Starting from the prefolded hinge, deployment tests were carried out by attaching a pair of tube holders to the apparatus previously used by Seffen and Pellegrino to study the behaviour of tape springs. ${ }^{9}$ This apparatus consists of two small gear boxes, one fixed to a rigid base and the other mounted on a linear bearing, supporting hollow strain-gauged shafts. The ends of the tube hinge are attached to the shafts, and this allows us to measure the applied end torques for any imposed end rotation.

First the strain gauge readings were set to zero in the deployed, unstressed configuration shown in Figure 3 . Then the tube hinge was flattened in the middle and fully folded by rotating the two ends by equal amounts. Subsequently, the ends were rotated back in small steps while keeping the end moments roughly equal; the rotations and moments were recorded at each step.

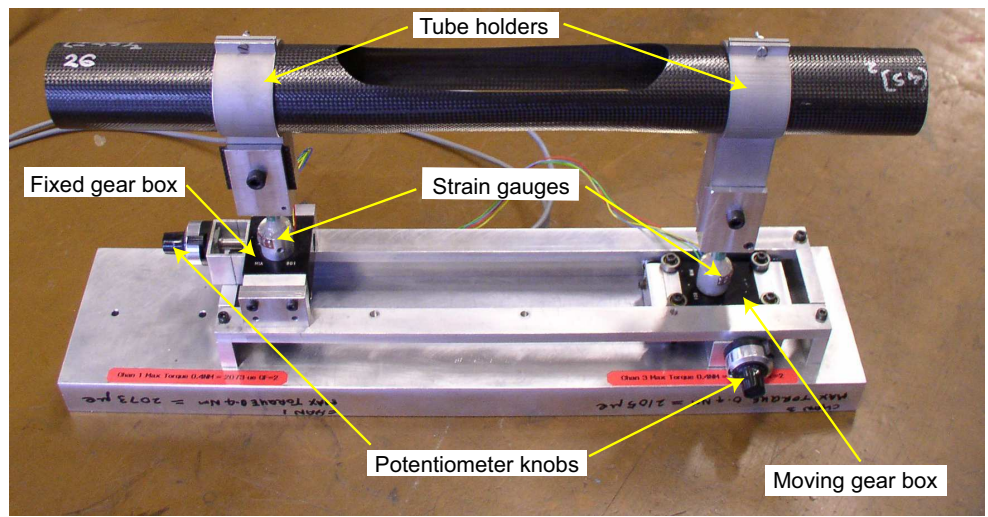

Figure 3. Setup for quasi-static deployment test.

At the end of the test the moment-rotation response of the tube hinge is obtained by computing at each step the deployment angle, defined as the difference between the end rotations, and the corresponding moment. Depending on the test method, two different moment-rotation relationships were obtained, see Figure 4. In the first method both potentiometer knobs were rotated simultaneously to a desired amount and then the fixed end knob was adjusted to equalize the end moments. This method leads to a negative moment for angles around $110^{\circ}$. In the second method the free end was rotated first and then the fixed end was adjusted to equalize the end moments. This method leads to an approximately constant bending moment until the final snap back.

It is believed that this difference in responses is due to the particular tube hinge design that has been chosen; the slot is too long and hence the position of the fold is not tightly controlled. Because the first test method leads to larger differences between the end moments, we will take the second test method to be more representative of the behaviour of the hinge. 


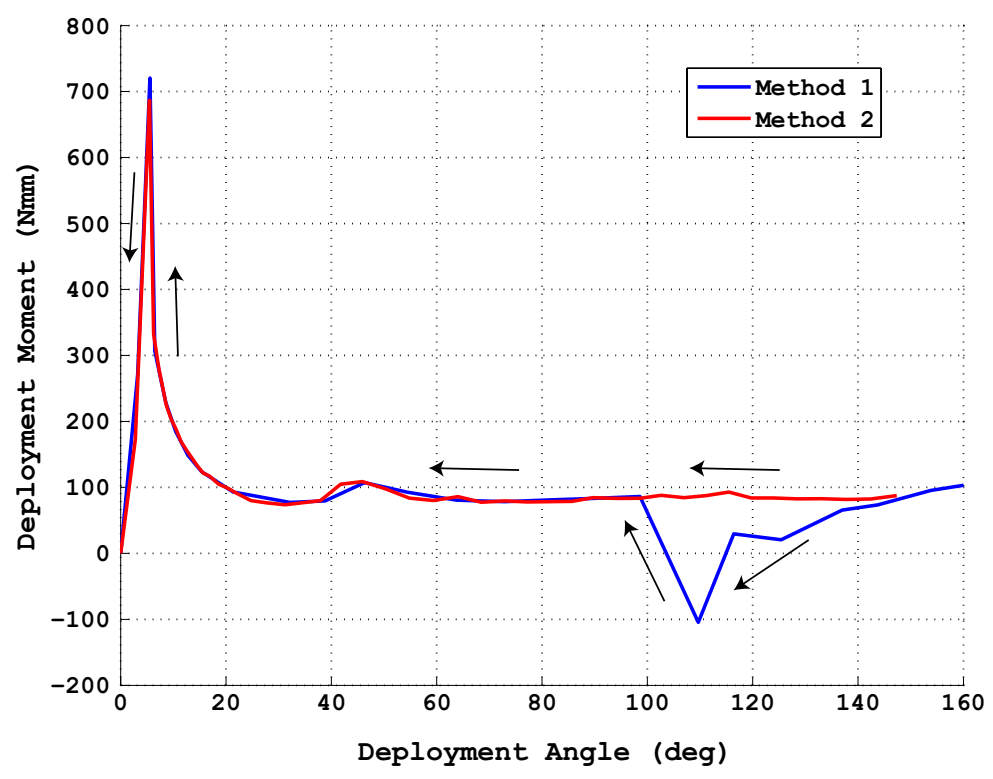

Figure 4. Deployment moment-rotation relationships measured by means of equal end rotation increments (method 1) or by increasing one rotation and then the other (method 2 ).

\section{Material Model}

Classical laminate theory does not provide accurate models for thin laminates made of woven prepregs. ${ }^{10}$ Hence, the composite material has been modeled as a linear-elastic thin plate whose properties are defined by the homogenization technique introduced by Kueh and Pellegrino ${ }^{11}$ for single-ply triaxial weave.

A representative unit cell is shown in Figure 5. In the case of two-ply laminates, the relative position of the plies is significant, ${ }^{12}$ we have assumed the extreme case where the tows are fully in phase. Each tow is modelled as a wavy beam of uniform cross-section, Figure 6, where the tow properties are defined in Table 1 and the cross-section is modelled as a $1.12 \mathrm{~mm}$ wide by $0.055 \mathrm{~mm}$ thick rectangle (based on measurements from micrographs of the cured laminate). The thickness of the rectangular section is denoted by $t$.

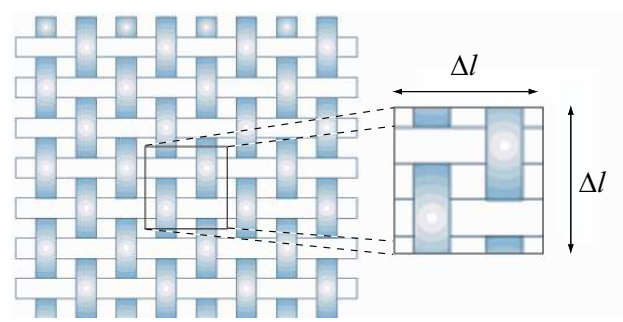

Figure 5. Representative unit cell for a single ply of plain weave composite.

Each tow consists of 32 B32 beam elements. The tow cross-overs are modeled using multi-point constraints and similarly the tow end nodes are tied by multi-point constraints to eight dummy boundary nodes that lie in the mid-plane of the composite.

The entries of the ABD matrix are computed using Virtual Work, after carrying out six separate ABAQUS/Standard analyses, each corresponding to a unit amplitude of the six deformation variables (midplane strains and curvatures). See Kueh and Pellegrino ${ }^{11}$ for details. 


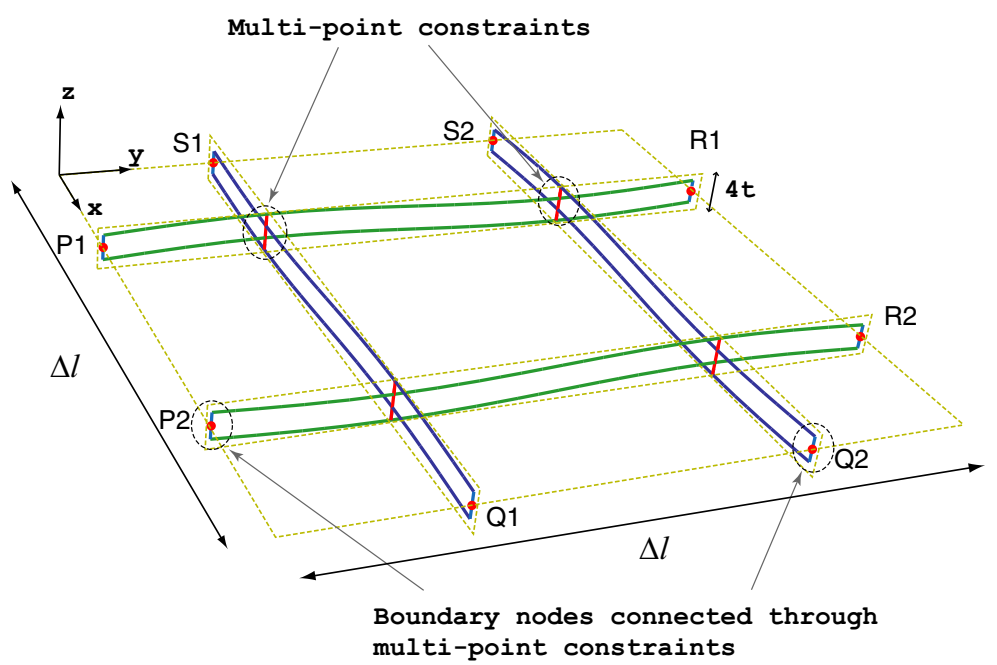

Figure 6. Model for unit cell of two-ply laminate.

\begin{tabular}{|l|c|}
\hline \hline Material Properties & Value \\
\hline Longitudinal stiffness, $E_{1}\left(\mathrm{~N} / \mathrm{mm}^{2}\right)$ & 159,520 \\
Transverse stiffness, $E_{2}=E_{3}\left(\mathrm{~N} / \mathrm{mm}^{2}\right)$ & 11,660 \\
Shear stiffness, $G_{12}=G_{13}\left(\mathrm{~N} / \mathrm{mm}^{2}\right)$ & 3,813 \\
In-plane Shear stiffness, $G_{23}\left(\mathrm{~N} / \mathrm{mm}^{2}\right)$ & 3,961 \\
Poisson's ratio, $\nu_{12}=\nu_{13}$ & 0.27 \\
Poissons ratio, $\nu_{23}$ & 0.472 \\
\hline
\end{tabular}

Table 1. Properties of T300-1k/913 tow 
The resulting constitutive relationship for the plate is

$$
\left\{\begin{array}{l}
N_{x} \\
N_{y} \\
N_{x y} \\
-- \\
M_{x} \\
M_{y} \\
M_{x y}
\end{array}\right\}=\left(\begin{array}{ccccccc}
9102 & 2877 & 0 & \mid & 0 & 0 & -0.5 \\
2877 & 9102 & 0 & \mid & 0 & 0 & 0.5 \\
0 & 0 & 112 & \mid & 0 & 0 & 0 \\
-- & -- & -- & -- & -- & -- & -- \\
0 & 0 & 0 & \mid & 45.4 & 0 & 0 \\
0 & 0 & 0 & \mid & 0 & 45.4 & 0 \\
-0.5 & 0.5 & 0 & \mid & 0 & 0 & 0.6
\end{array}\right)\left\{\begin{array}{c}
\epsilon_{x} \\
\epsilon_{y} \\
\epsilon_{x y} \\
-- \\
\kappa_{x} \\
\kappa_{y} \\
\kappa_{x y}
\end{array}\right\}
$$

where $N$ denotes force per unit length, $M$ moment per unit length, $\epsilon$ mid-plane strain and $\kappa$ mid-plane curvature. The subscripts $x$ and $y$ represent the global directions and the units are $\mathrm{N}$ and $\mathrm{mm}$.

This matrix can be rotated $45^{\circ}$ to the element reference axes (in the longitudinal and hoop directions for the tube hinge) to obtain

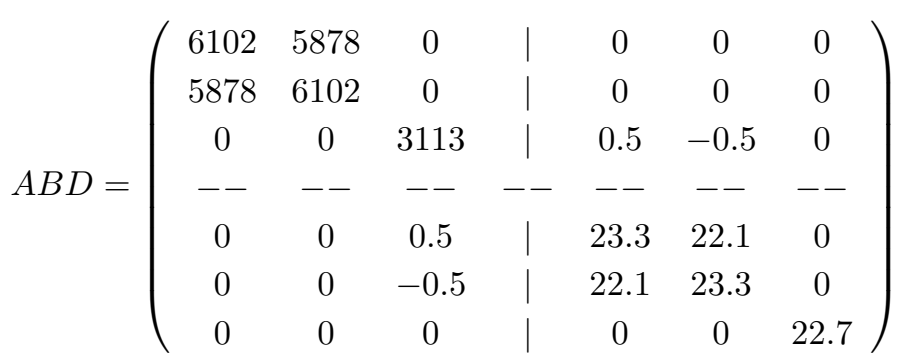

\section{Finite Element Model}

Two different models of the tube hinge were created. The first model consisted of a $220 \mathrm{~mm}$ long, slotted cylindrical surface with end sections rigidly connected to reference nodes subjected to certain prescribed boundary conditions. This model consisted of 2831 nodes and 2645 four-node, reduced integrated shell elements S4R (with the enhanced hourglass control option switched on). The minimum element length in the mesh was approximately $3 \mathrm{~mm}$. The second, more refined model — see Figure 7 - included the full length of the actual tube hinge, $400 \mathrm{~mm}$, and took into account both the elastic compliance of the holders attaching the tube to the testing machine and the clearance at the interface between the tube and the holders. The complete model consisted of 4354 nodes and 4200 S4R elements with approximate element length of $3 \mathrm{~mm}$.

In order to simulate the folding of the tube hinge the two reference nodes, $\mathrm{A}$ and $\mathrm{B}$, at either end were connected to a dummy node, $\mathrm{C}$, using the ABAQUS command ${ }^{*} E Q U A T I O N$. Then the following boundary conditions were prescribed. All degrees of freedom of node A were restrained, except the rotation about the global X-axis. Node B was only allowed to translate along the global Z-axis and to rotate about the global $\mathrm{X}$-axis. To bend the tube hinge with equal end moments the rotational degree of freedom of nodes $\mathrm{A}$ and $\mathrm{B}$ was constrained as follows

$$
\theta_{X}^{A}-\theta_{X}^{B}=\theta_{X}^{C}
$$

where $\theta_{X}$ denotes the rotation about the global X-axis . $\theta_{X}^{C}$ was incremented from $0^{\circ}$ to $170^{\circ}$ over a prescribed time interval using the ABAQUS/Explicit command *AMPLITUDE, DEFINITION=SMOOTH $S T E P$. This prescribes a fifth-order polynomial time variation with first and second derivatives equal to zero at the beginning and end of the time interval.

The elastic properties of the shell were defined with the *SHELL GENERAL SECTION parameter by assigning the ABD matrix obtained in Section III, defined with respect to a cylindrical coordinate system, 1, 2, 3 respectively radial, circumferential and longitudinal.

The GENERAL CONTACT feature was assigned to the entire model by specifying CONTACT INCLUSIONS, ALL EXTERIOR. With this option ABAQUS/Explicit automatically defines potential contact surfaces around the whole tube surface. The interaction between two CFRP surfaces was defined as frictionless, as the smooth finish of the tube does not provide much sliding resistance. However, in the second model the interaction between the tube hinge and the holders was defined as rough.

The first fundamental mode of vibration of the tube hinge was estimated with a Linear Perturbation Frequency analysis run in ABAQUS/Standard. This gave $0.011 \mathrm{~s}$ for the first model. Hence, the folding 


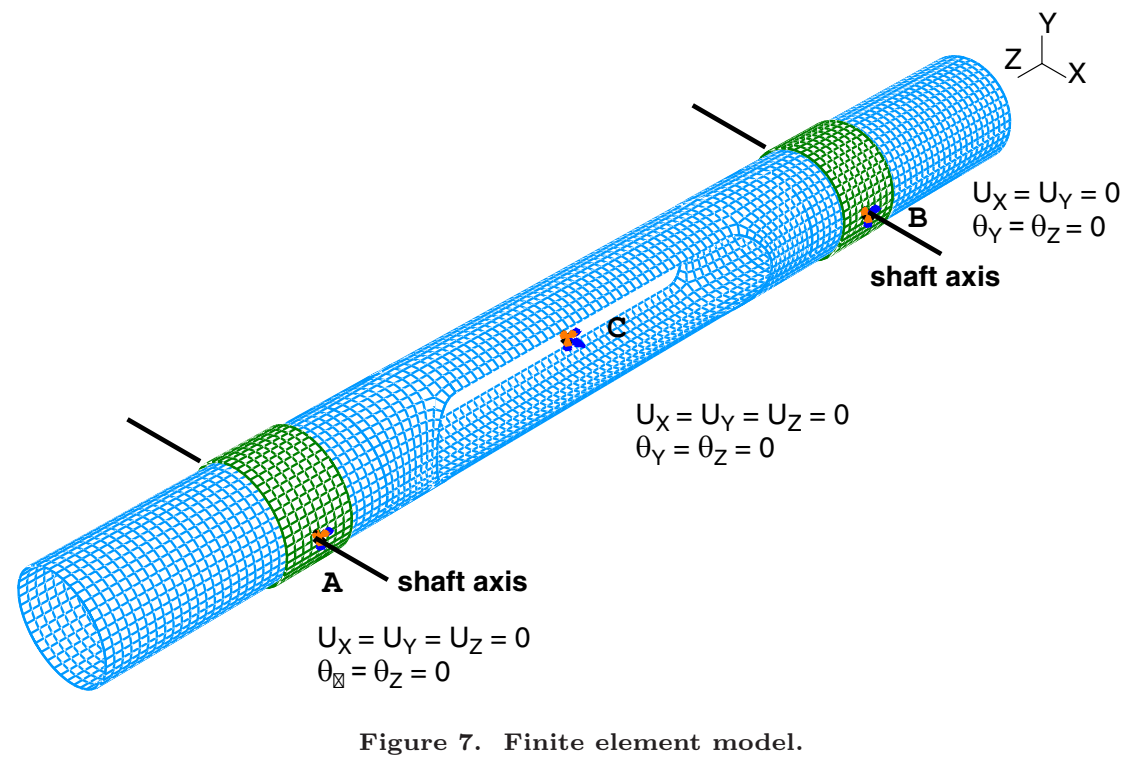

simulation was initially carried out for $0.1 \mathrm{~s}$, however it was found at certain points during the simulation the kinetic energy was higher than $10 \%$ of the strain energy and hence the simulation time had to be increased to $3 \mathrm{~s}$. The double precision solver was used. The anti-aliasing filter was used to filter out the high frequency response.

The time increment during the simulation is automatically set to

$$
\Delta t=\frac{2}{\omega_{\max }}\left(\sqrt{1+\xi^{2}}-\xi\right)
$$

where $\omega_{\max }$ is the highest eigenvalue in the current configuration and $\xi$ is the fraction of critical damping in the highest mode.

The moment-rotation relationship obtained from the two models is shown in Figure 8. The first model was rerun with the fully integrated S4 shell elements and the results are also shown in the figure. A comparison of these results leads to the conclusion that the reduced integration elements lead to a lower peak moment.

The fully folded configuration obtained at the end of the folding simulations described above can be used as the starting configuration for a deployment simulation. However, before starting the deployment simulation the kinetic energy in the folded structure has to be dissipated. This can be done in several ways; the easiest way is to run an additional simulation step with the ends held stationary. Next, to simulate deployment the hinge was rotated back to its original configuration, by assigning opposite variation to $\theta_{X}^{C}$ over a prescribed simulation period. Again, a smooth variation of the angle over time was prescribed, to minimize accelerations.

Figure 9 shows the simulation results obtained from a full folding and deployment cycle. The momentrotation response was recorded at every $1 \mathrm{~ms}$ and an anti-aliasing filter was applied to the results. The most striking result is that the peak deployment moment is less than half the peak moment required to fold the tube hinge. This is not surprising as this behavior is characteristic of structures with an unstable post-buckling equilibrium path. It is already known that tape spring structures behave in this way, ${ }^{9}$ but having established that tube hinges share this feature is a result with important practical implications.

Figure 10 shows the corresponding variation of the energy terms during this simulation. Here the internal energy and energy balance are calculated from

$$
\begin{gathered}
E_{I E}=E_{S E}+E_{A E} \\
E_{b a l}=E_{I E}+E_{K E}+E_{V D}-E_{W K}
\end{gathered}
$$

where $E_{I E}=$ internal energy, $E_{S E}=$ strain energy, $E_{A E}=$ artificial energy, $E_{b a l}=$ energy balance, $E_{K E}=$ kinetic energy, $E_{V D}=$ viscous dissipation and $E_{W K}=$ work done. Note that, to define more accurately the 


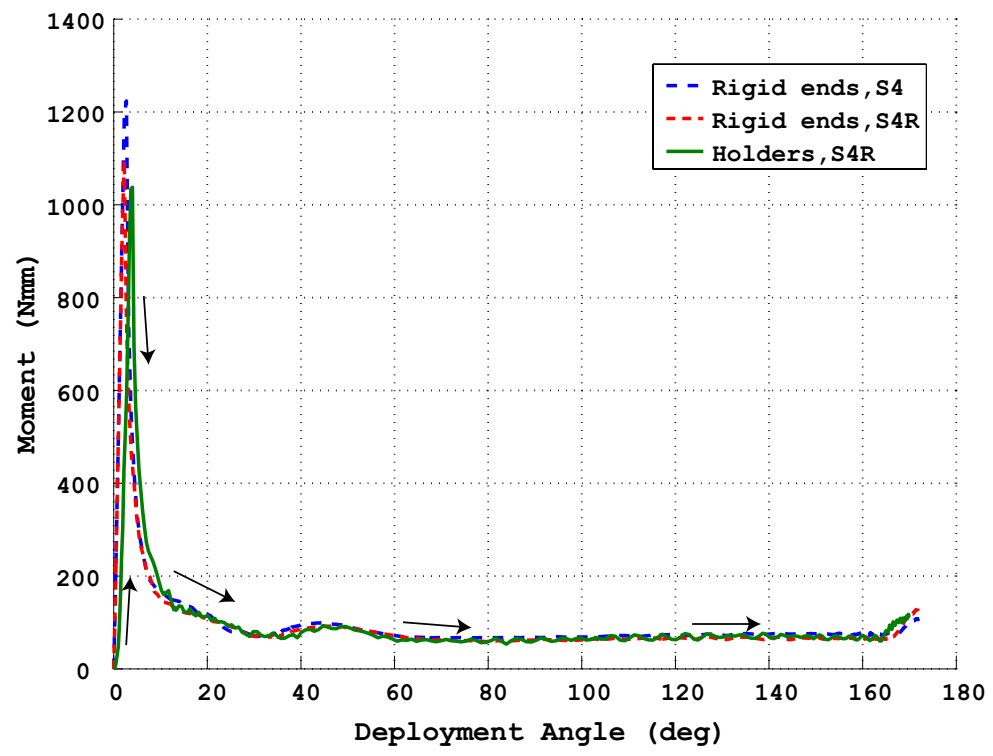

Figure 8. Moment-rotation relationships (folding only) for different boundary conditions (rigid ends vs. compliant holders) and shell elements (S4 vs. S4R); each simulation was run for $3 \mathrm{~s}$.

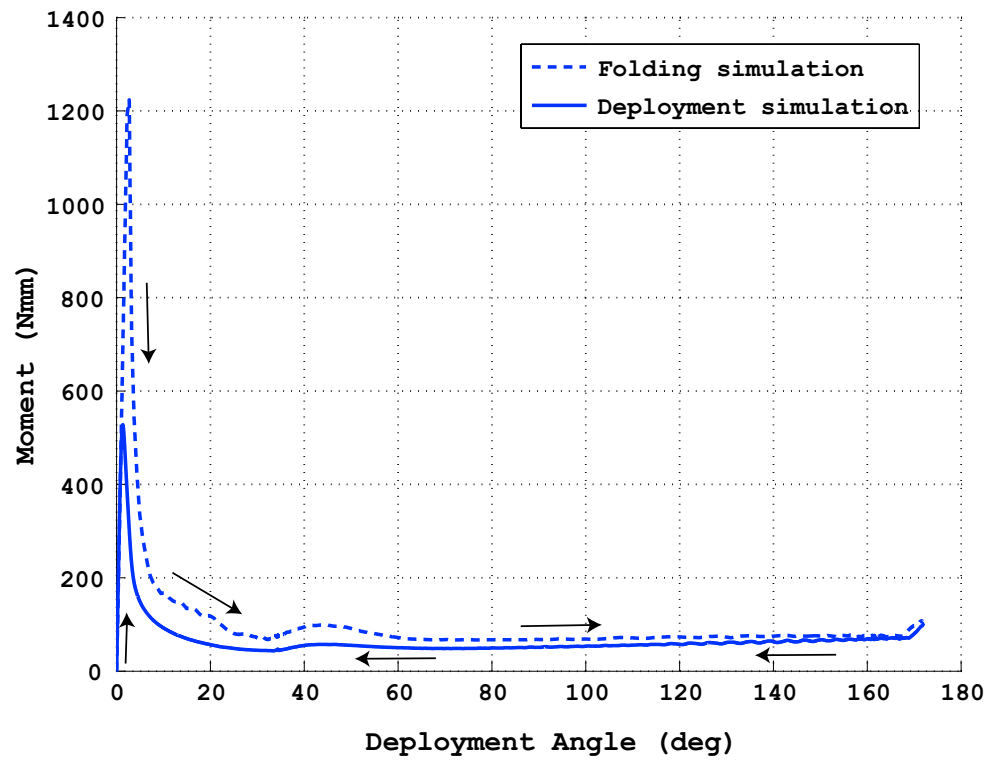

Figure 9. Complete moment-rotation relationship obtained from folding and deployment simulation cycle, using S4 elements. See also Fig. 10. 


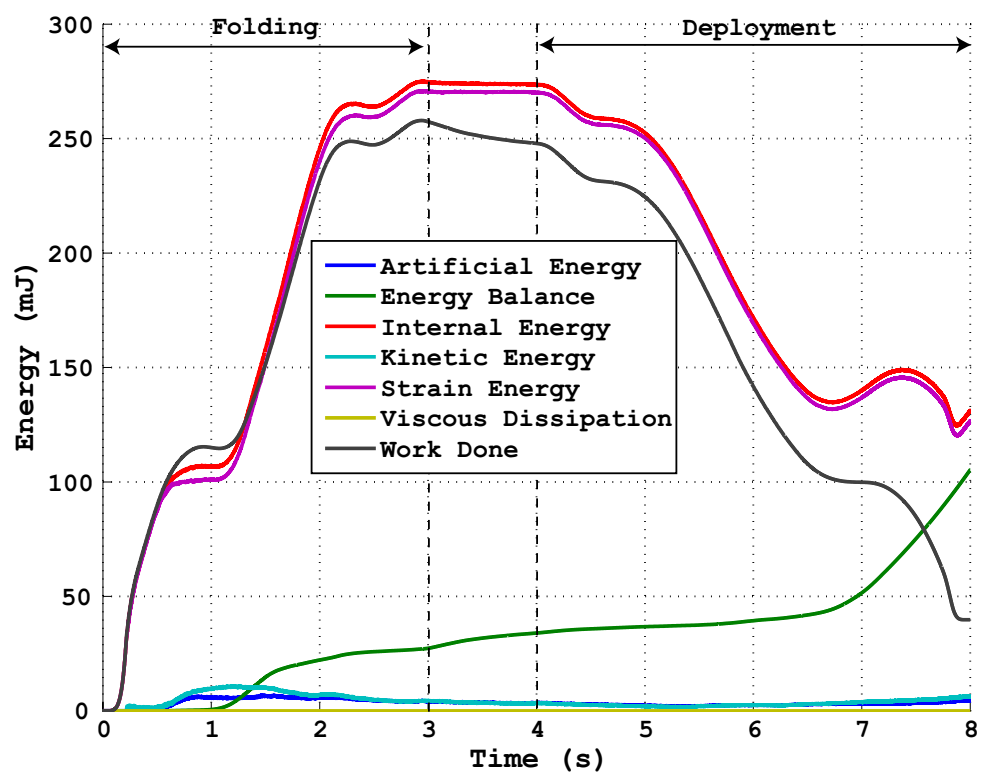

Figure 10. Energy variation corresponding to moment-rotation relationship in Figure 9.

time variation of the deployment angle at some intermediate steps, each simulation has been divided into three separate steps.

The plot indicates that between $1 \mathrm{~s}$ and $8 \mathrm{~s}$ there is a positive energy balance. This is a surprising result that we cannot explain at present. Note that the kinetic energy is always less than $10 \%$ of the strain energy.

Figure 11 show the results of a simulation using a mesh of S4R elements. In this case the work done is almost always higher than the strain energy in the structure, which would suggest that the latter simulation is more accurate. However, see the comment in Section VIII.

\section{Sensitivity Analyses}

Several sensitivity analyses were carried out to refine the folding simulation, for the mesh of S4R elements. It is planned to extend this study to the deployment simulations, in future.

The sensitivity to the time increment size was analyzed by running folding simulations for deployment angles from $0^{\circ}$ to $35^{\circ}$, which corresponds to the most non-linear portion of the moment-rotation response, with five different scaling factors, 0.10, 0.25, 0.50, 0.75, 0.80, for the ABAQUS/Explicit default time increment, Eq. 4. It was observed that the moment-rotation relationship is practically unaffected by this change.

The sensitivity to the mesh density was investigated by almost doubling the number of elements (from 2831 nodes and 2645 S4R elements to 4357 nodes and 4092 S4R elements), and hence reducing the minimum element length from $3 \mathrm{~mm}$ to about $2 \mathrm{~mm}$. This change, that left all results practically unchanged, automatically affects the time step computed by ABAQUS/Explicit.

\section{Folded Configuration}

The mid-plane strains in the directions of the fibres in the fully folded configuration were evaluated. This was done by transforming the mid-plane strains computed by ABAQUS/Explicit with a standard strain transformation

$$
\begin{aligned}
& \epsilon_{+45}=\cos ^{2} \phi \epsilon_{1}+\sin ^{2} \phi \epsilon_{2}+\cos \phi \sin \phi \gamma_{12} \\
& \epsilon_{-45}=\cos ^{2} \phi \epsilon_{1}+\sin ^{2} \phi \epsilon_{2}-\cos \phi \sin \phi \gamma_{12}
\end{aligned}
$$

where $\phi=45^{\circ}$.

Figure 12 compares the mid-plane strain distributions in the +45 fibres, for the fully folded configurations obtained from simulations using S4R and S4 elements. 


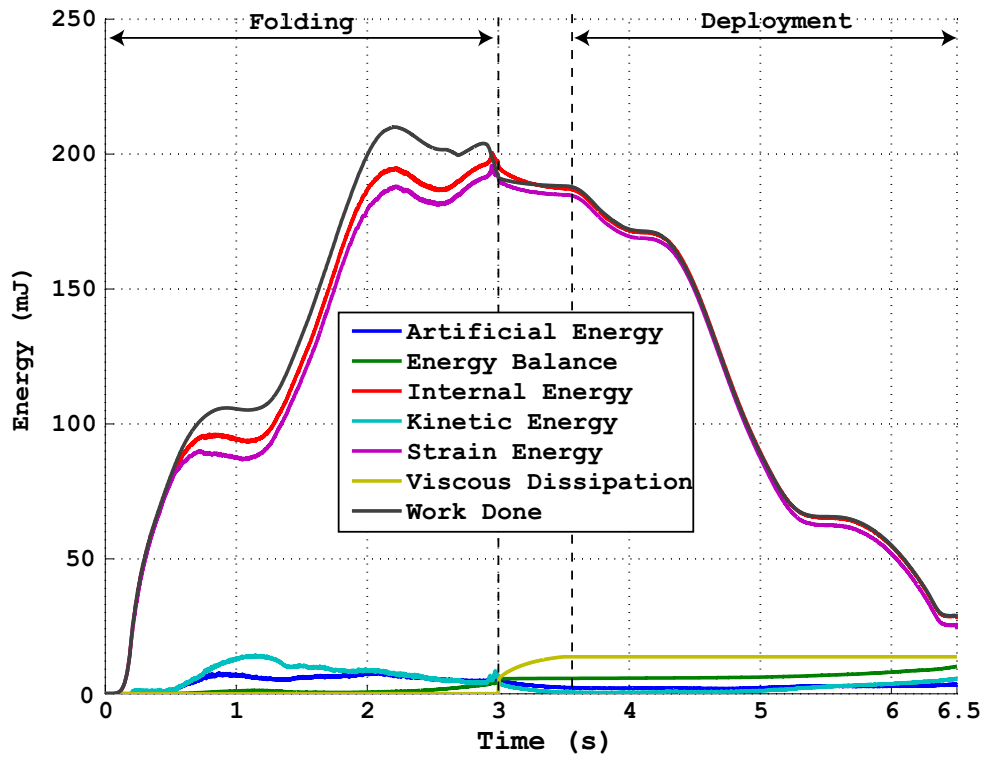

Figure 11. Energy variation for S4R mesh with viscosity.

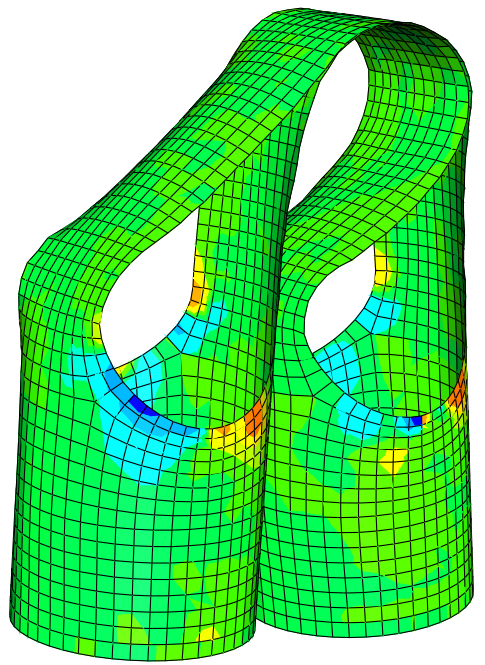

(a)
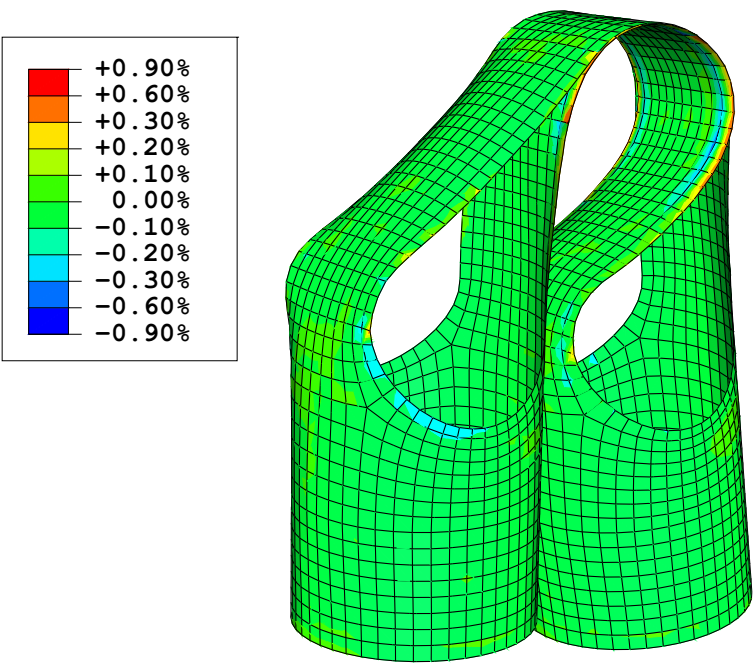

(b)

Figure 12. Mid-plane strains in $+45^{\circ}$ fibres: (a) S4R elements (b) S4 elements 
Following Yee and Pellegrino, ${ }^{10}$ we are particularly interested in the maximum curvatures in the laminate, because the maximum curvature provides the most relevant measure of how close the folded structure is to failure. For clarity we will consider the longitudinal and transverse curvatures. Figure 13 and 14 compare the longitudinal and transverse curvature variations with S4R elements and S4 elements respectively.

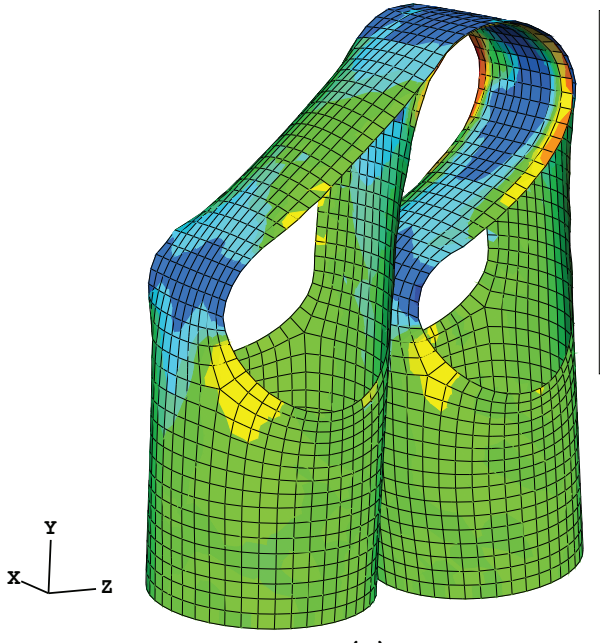

(a)
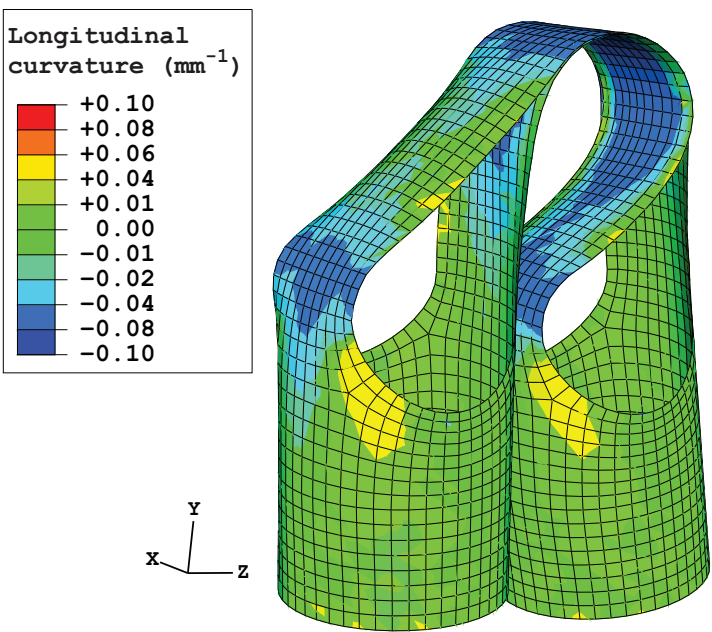

(b)

Figure 13. Longitudinal curvature changes: (a) S4R elements (b) S4 elements

The maximum amplitude of the mid-plane strain predicted with the two types of elements, Figure 12, are similar but the locatin is different. The highest fibre strain magnitudes, of up to $0.9 \%$ occur in regions of small curvature changes. Since this is value is well below the failure strain of T300 carbon fibre (around $1.5 \%^{11}$ ) it is clear that the tube hinge is safe.

The distributions of curvature changes predicted with the two types of elements, shown in Figures 13 and 14, are also similar. The largest curvature change is less than $0.13 \mathrm{~mm}^{-1}$ and occurs in a region of low mid-plane strain. According to Reference ${ }^{10}$ (which however used a different resin) this curvature is well below the failure value for biaxial curvatures in any direction, hence showing that the tube hinge is safe.

\section{Comparison of Experiment and Simulations}

Figure 15 shows a comparison between the deformed configurations of the tube hinge that are observed during quasi-static deployment, in the testing rig, with snapshots from the finite element simulation. This qualitative comparison shows very good agreement between the position and overall configuration of the regions of localized deformation. However it should be noted that the fully deployed configuration obtained from the simulation, on the bottom right of the figure, did not fully recover the original configuration.

The hinge appears to have fully snapped back, but the ends are not completely aligned. It is interesting to compare the final, fully deployed shapes obtained from simulations with S4R and S4 elements, shown in Figure 16. Although in Section IV the former simulation appeared more accurate on the basis of energetic considerations, the latter has produced lower residual strains at the end.

Figure 17 shows a comparison between the deployment moment-rotation relationship measured experimentally with the simulated response using S4 elements. Overall, the simulation has fully captured the experimentally observed behavior. However, the measured peak moment at snap back was $690 \mathrm{Nmm}$ at a rotation of around $5.5^{\circ}$ whereas the simulation gave a value of $530 \mathrm{Nmm}$ at $1.5^{\circ}$. Also, the measured steady- 


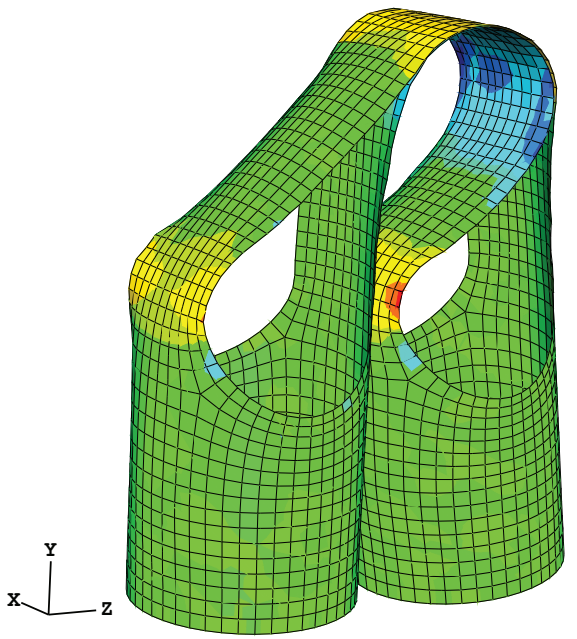

(a)

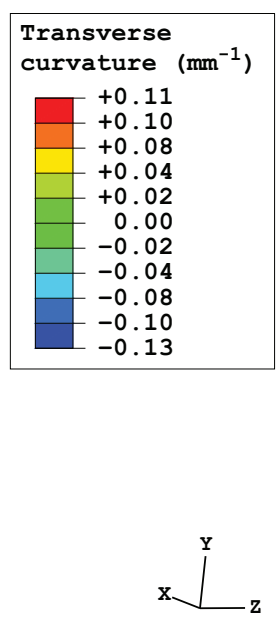

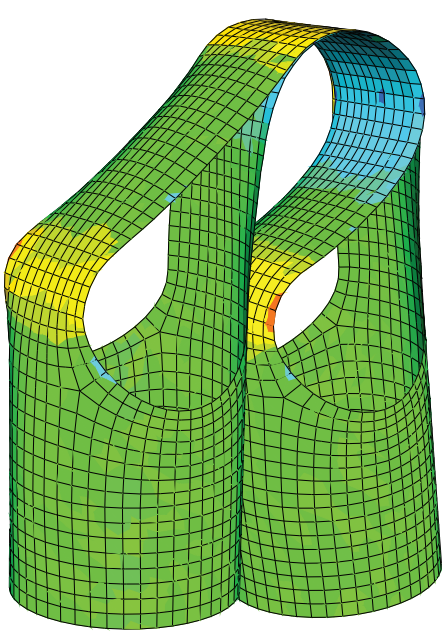

(b)

Figure 14. Transverse curvature changes: (a) S4R elements (b) S4 elements

state deployment moment had an average value of $85 \mathrm{Nm}$ whereas our simulation gave around $60 \mathrm{Nmm}$. Lastly, the measured stiffness of the hinge after snap back was less than half of the predicted value.

\section{Discussion}

In this paper we have presented a detailed study of the folding and deployment of a slotted tube hinge made from a two-ply laminate of carbon-fibre reinforced plastic. We have constructed a physical model of a particular version of this hinge and used this model to carry out quasi-static deployment tests, starting from the fully folded configuration. Our main contribution is that we have been able to capture this process through a finite element simulation. The first stage in this simulation was to generate the fully folded, strained configuration of the hinge; this was done by applying equal and opposite moments on either end of the hinge, whereas in reality one would pinch the hinge in the middle to reduce the peak moment required to fold hinge. The second stage in the simulation was to gradually decrease the relative rotation between the ends until it became zero. This process has captured both the steady-state moment part of the deployment, during which a localized fold can be seen in each of the tape springs that make up the hinge, as well as the snap back to the straight configuration.

By analysing the in-plane strains and out-of-plane curvatures in the folded configuration we have confirmed that the particular hinge design that has been studied could be folded without permanent damage.

Next stages in this research will involve a more detailed analysis of the variation of the various energy components that contribute to the ABAQUS/Explicit solution, a sensitivity study of the quasi-static simulation results to mesh design and solution control parameters, and a further investigation of the residual strains that have been found in the fully deployed configuration. Furthermore, we plan to validate experimentally and possibly refine the elastic constitutive model for the woven laminate and the elastic compliance model for the bending rig used for the deployment test. Lastly, we plan to investigate the dynamic deployment behavior of tube hinges.

\section{Acknowledgments}

We thank Dr Ahmad Kueh and Dr Michael Sutcliffe for helpful discussions. We are grateful to Mr J. Ellis (Hexcel, UK) for providing materials. HM thanks the Cambridge Commonwealth Trust for financial support. 


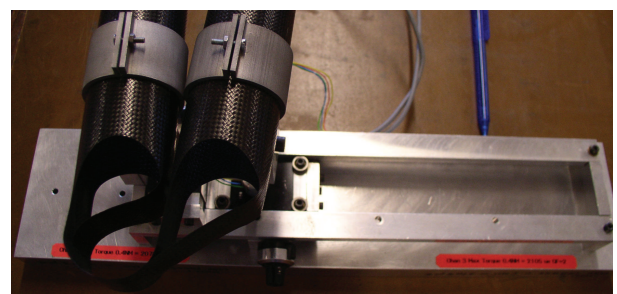

$171^{0}$
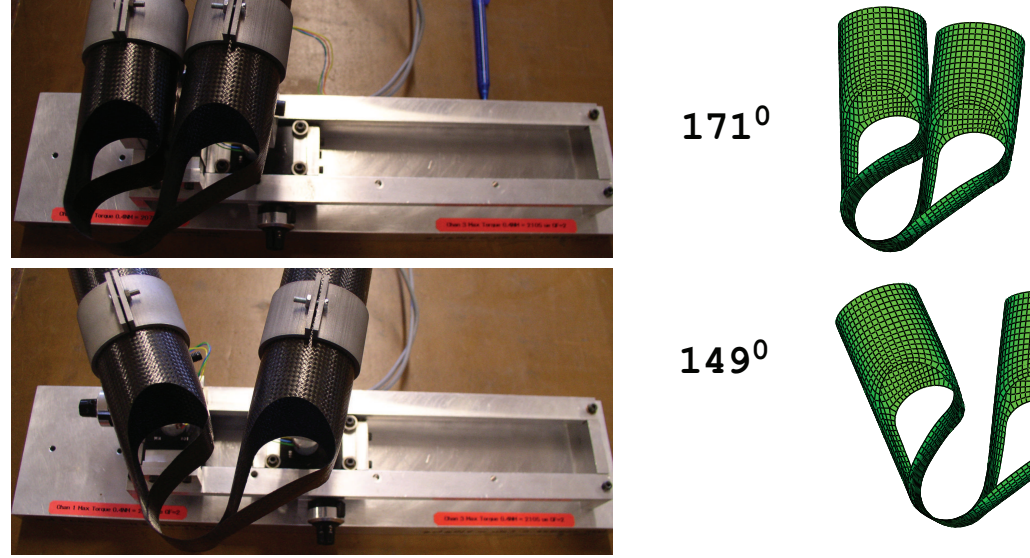

$149^{\circ}$
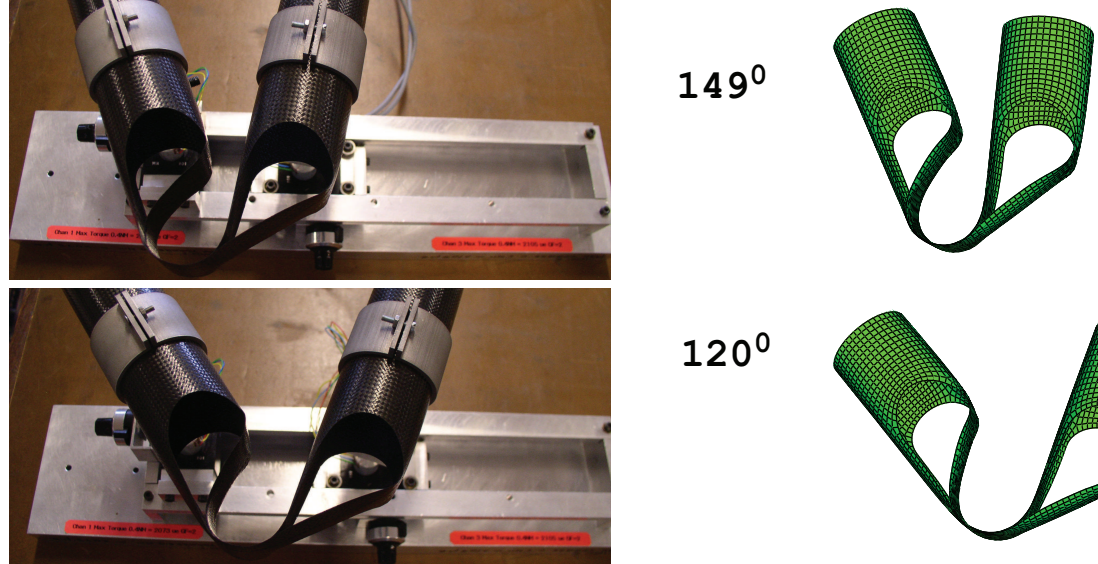

$120^{\circ}$
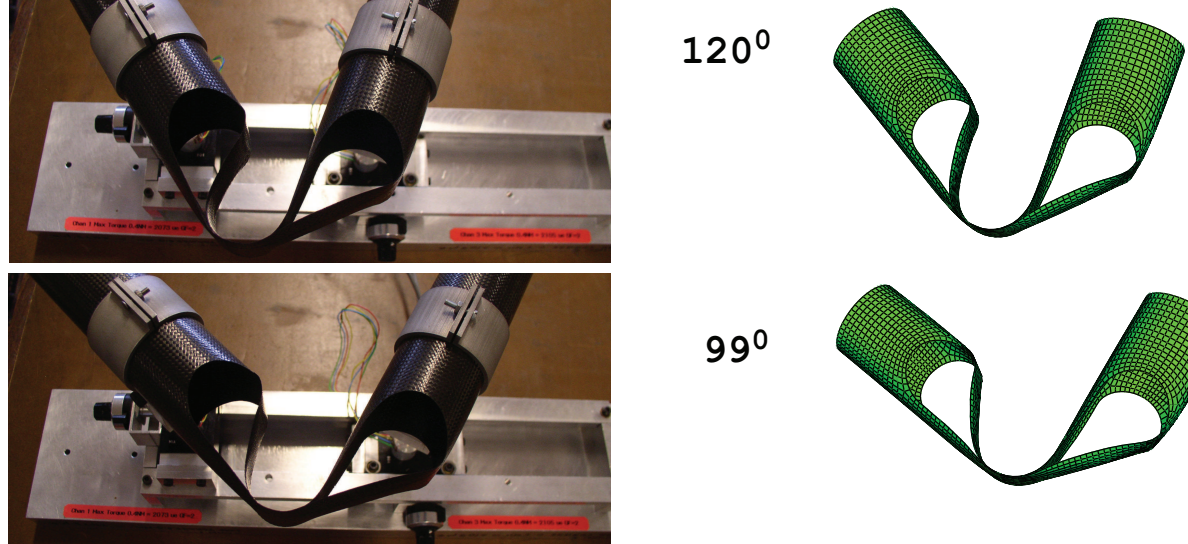

$99^{\circ}$
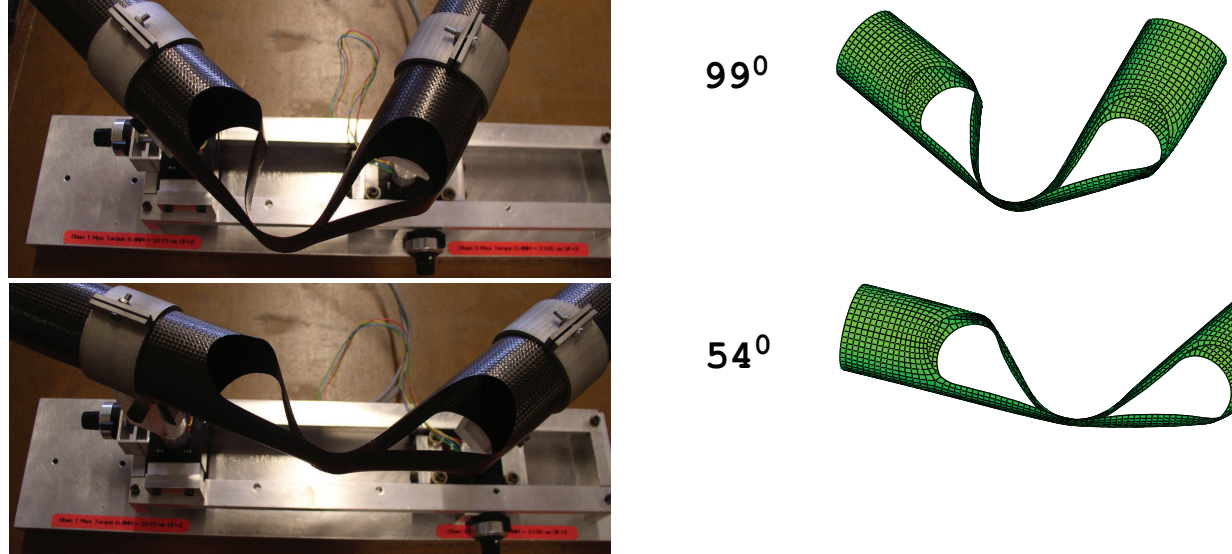

$54^{0}$
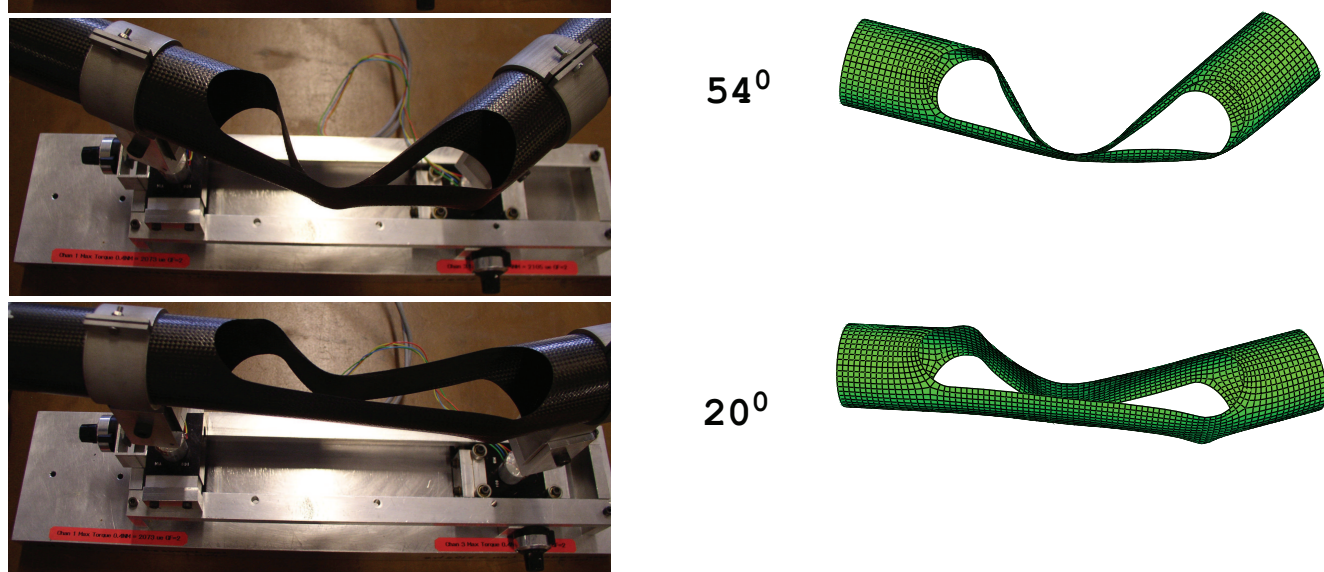

$20^{0}$
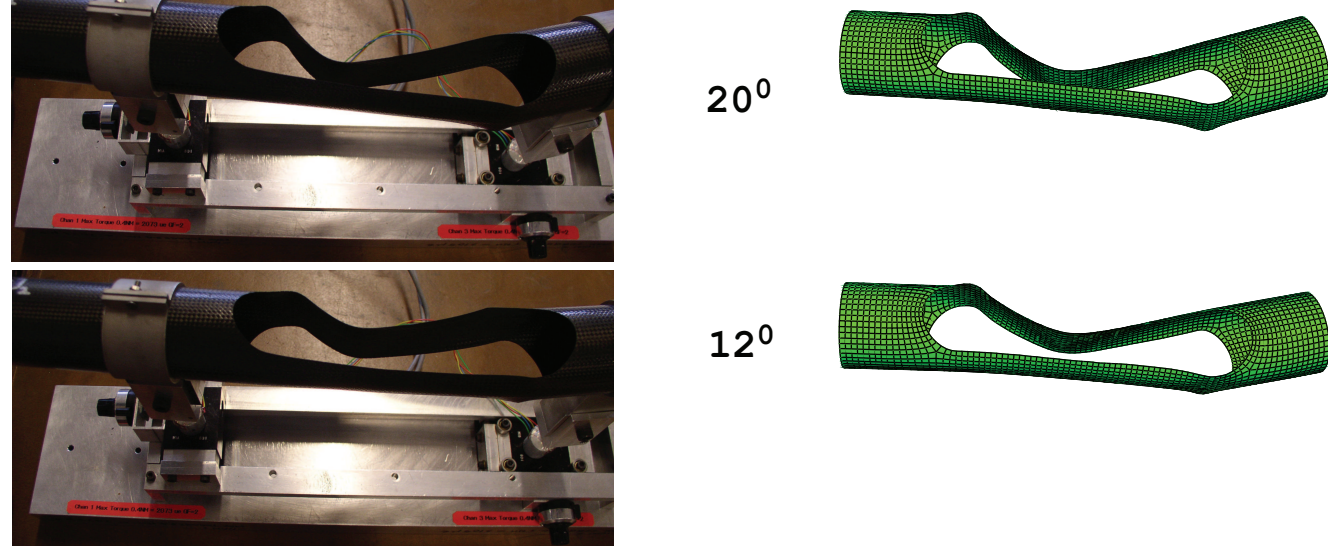

$12^{0}$
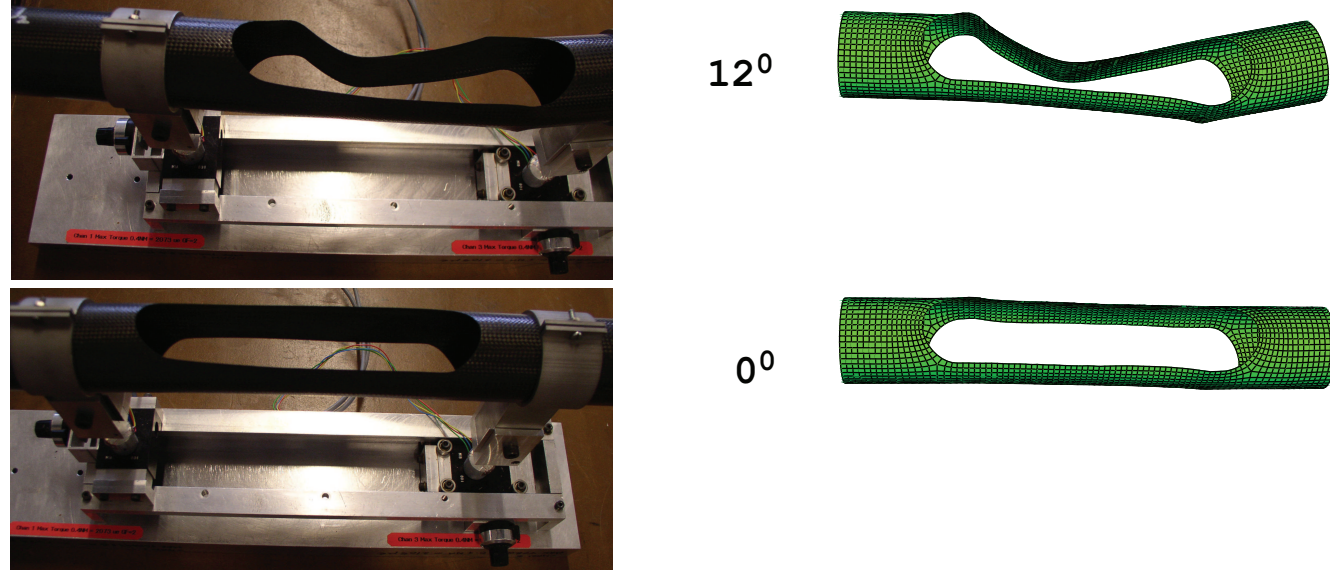

$0^{0}$

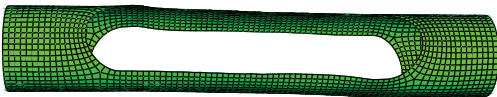

Figure 15. Comparison of hinge configurations during deployment (simulation is with S4 elements) for different values of the deployment angle.

13 of 15 


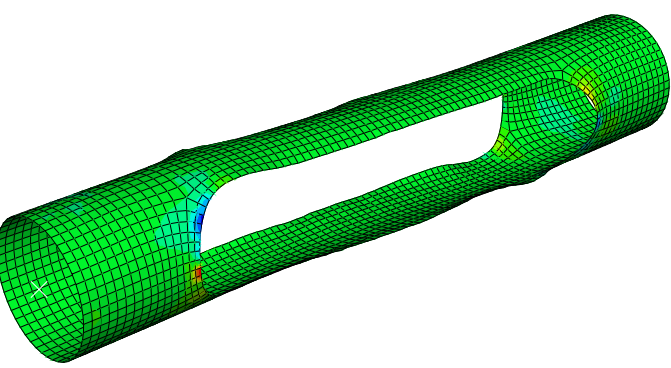

(a)

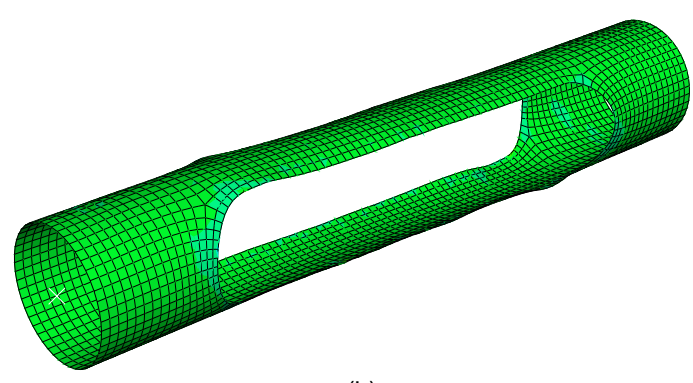

(b)

Figure 16. Fully deployed configurations (a) S4R elements (b) S4 elements.

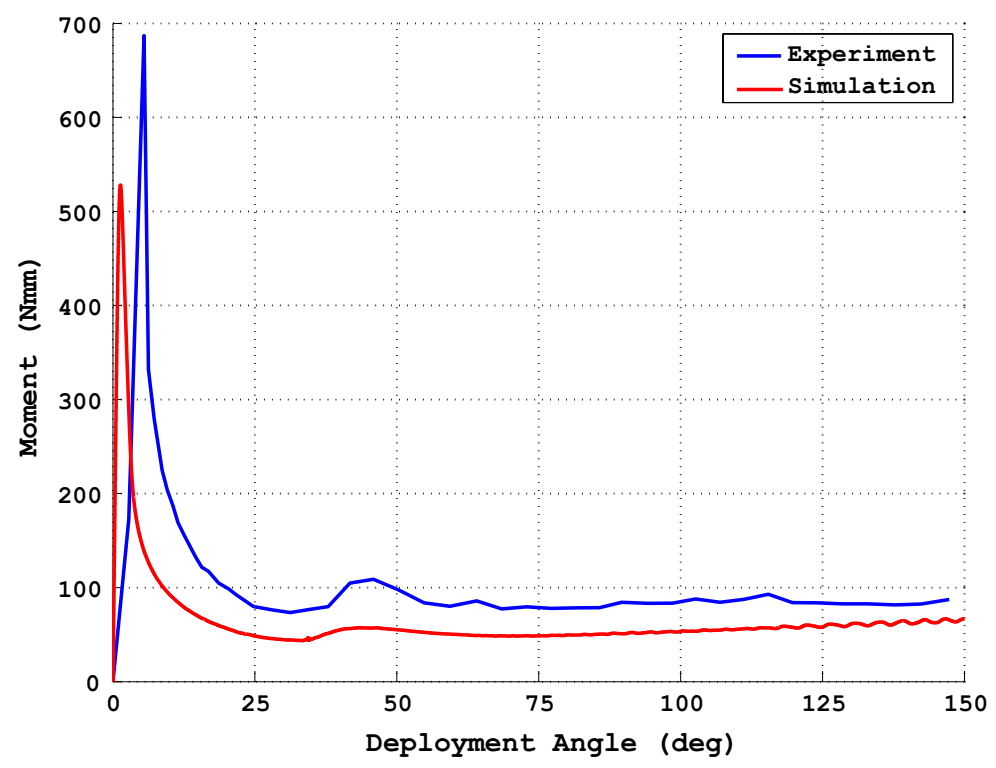

Figure 17. Comparison of deployment moment-rotation relationships. 


\section{References}

${ }^{1}$ Mobrem, M. and Adams, D.S., "Analysis of the lenticular jointed MARSIS antenna deployment," 47 th AIAA/ASME/ASCE/AHS/ASC Structures, Structural Dynamics, and Materials Conference, AIAA-2006-1683, Newport, Rhode Island, 1-4 May 2006.

${ }^{2}$ O. Soykasap, A. Watt and Pellegrino, S., "New deployable reflector concept," 45th AIAA/ASME/ASCE/AHS/ASC Structures, Structural Dynamics, and Materials Conference, AIAA 2004-1574, Palm Springs, California, 19-22 Apr 2004.

${ }^{3}$ Jorgensen, J., Louis, E., Hinkle, J., and Silver, M., "Dynamics of an elastically deployable solar array: ground test model validation," 46th AIAA/ASME/ASCE/AHS/ASC Structures, Structural Dynamics, and Materials Conference, AIAA 2005-1942, Austin, Texas, 18-21 April 2005.

${ }^{4}$ Soykasap, O., Pellegrino, S., Howard, P. and Notter, M., "Tape spring large deployable antenna," 47 th AIAA/ASME/ASCE/AHS/ASC Structures, Structural Dynamics, and Materials Conference, AIAA 2006-1601, Newport, Rhode Island, 1-4 May 2006.

${ }^{5}$ Adams, D.S. and Mobrem, M., "MARSIS antenna flight deployment anomaly and resolution," 47th AIAA/ASME/ASCE/AHS/ASC Structures, Structural Dynamics, and Materials Conference, AIAA 2006-1684 1 - 4 May 2006, Newport, Rhode Island.

${ }^{6}$ Yee, J. C. H. and Pellegrino, S., "Composite tube hinges," Journal of Aerospace Engineering, Vol. 18, No. 4, 2005, pp. $224-231$

${ }^{7}$ SIMULIA, ABAQUS/Explicit Version 6.7, Providence, RI

${ }^{8}$ Mallikarachchi, H.M.Y.C., "Numerical simulation and experimental validation of thin-walled deployable structures," First year report, University of Cambridge, 2007.

${ }^{9}$ Seffen, K. A. and Pellegrino, S., "Deployment dynamics of tape springs," Proceedings of the Royal Society of London Series A, Vol. 455, 1999, pp. 1003-1048.

${ }^{10}$ Yee, J. C. H. and Pellegrino, S., "Biaxial bending failure locus for woven-thin-ply carbon fibre reinforced plastic structures" 46th AIAA/ASME/ASCE/AHS/ASC Structures, Structural Dynamics, and Materials Conference, AIAA 2005-1811, Austin, Texas, 18-21 April 2005.

${ }^{11}$ Kueh, A. B. H. and Pellegrino, S., "ABD matrix of single-ply triaxial weave fabric composites," 48th AIAA/ASME/ASCE/AHS/ASC Structures, Structural Dynamics, and Materials Conference, AIAA-2007-2161, Honolulu, Hawaii, 23-26 April 2007.

${ }^{12}$ Soykasap, O., "Micromechanical models for bending behaviour of woven composites," 46th AIAA/ASME/ASCE/AHS/ASC Structures, Structural Dynamics, and Materials Conference, AIAA-2005-2264, Austin, Texas, 18-21 April 2005. 